\title{
Changes in RR and QT Intervals after Spontaneous and Respiratory Arousal in Patients with Obstructive Sleep Apnea
}

\author{
M Baumert ${ }^{1}$, J Smith ${ }^{1,2}$, P Catcheside ${ }^{2}$, DR McEvoy ${ }^{2}$, \\ D Abbott ${ }^{1}$, E Nalivaiko ${ }^{3}$ \\ ${ }^{1}$ The University of Adelaide, Adelaide, Australia \\ ${ }^{2}$ Repatriation General Hospital, Adelaide, Australia \\ ${ }^{3}$ Flinders University, Adelaide, Australia
}

\begin{abstract}
Obstructive sleep apnea (OSA) is a common disorder that has been associated with hypertension and ventricular arrhythmias. The aim of this study was to investigate changes in $R R$ and $Q T$ intervals after respiratory and spontaneous arousals in patients with OSA. We conducted overnight sleep studies in 20 patients. The mean and range of respiratory disturbance index were $49 \pm 28$ and [17-107], respectively. During stage 2 sleep, ECGs were analyzed over a period of 30 seconds. From the 187 arousals investigated (120 respiratory vs. 67 spontaneous) a significant number were associated with a RR interval shortening (64\%). Eighteen percent of the RR intervals were prolonged, with a significantly higher number during spontaneous arousals (13\% vs. 27\%). Similarly, QT intervals shortened after arousal (65\%). But there were no significant differences between spontaneous and respiratory arousals. In conclusion, spontaneous and respiratory arousals lead to changes in heart rate and ventricular repolarization.
\end{abstract}

\section{Introduction}

The obstructive sleep apnea (OSA) is a common disorder of breathing during sleep affecting more than $4 \%$ of men and $2 \%$ of women [1] that is characterized by repetitive partial or complete closure of the upper airway during sleep. Acute physiologic stress occurring during each obstructive episode leads to dramatic visceral changes, including marked blood gas disturbance/asphyxia; large negative intrathoracic pressure changes that increase cardiac pre- and afterload, surges in sympathetic neural activity, alterations in heart rate, surges in arterial blood pressure and cortical arousals. Obstructive events, which may occur hundreds of times a night, are thought likely to contribute to pre-morbid cardiovascular disease progression as well as causally to cardiovascular disease itself. Given the acute and repetitive nature of overnight cardiovascular stress associated with OSA, these events likely contribute to potentially fatal nocturnal cardiovascular events such as ventricular arrhythmias, myocardial infarction and stroke $[2,3]$.

Cardiac activity in OSA patients has been studied mainly based on RR interval changes (heart rate variability, HRV) as a maker of autonomic control. HRV has shown to be an independent predictor of cardiac mortality in different patient populations, including myocardial infarction (MI) [4], and congestive heart failure (CHF) [5]. It has been suggested that autonomic heart rate modulation is altered during sleep in patients with OSA [6].

In this study we investigated RR and QT interval changes associated with spontaneous and respiratory arousal in patients with OSA in order to assess possible instantaneous changes in the electric activity of the heart.

\section{Methods}

\subsection{Patients}

Overnight polysomnographic sleep studies were conducted in 20 subjects with suspected OSA (12 males). The average ages was $42 \pm 9$ years and the average BMI was $35 \pm 12 \mathrm{~kg} \cdot \mathrm{m}^{2}$. None of the patients had a craniofacial, metabolic or respiratory disorder, cardiovascular disease or diabetes. Further, none of the patients had insomnia or other forms of sleep disorders. All patients were nonsmokers.

\subsection{Overnight polysomnography}

Overnight polysomnography was recorded, using a Compumedics E series system (Australia). For sleep staging and arousal scoring standard surface electrodes were applied to the face and scalp, including two-channel electroencephalograms (EEG, C3-A2 and C4-A1), left and 
right electrooculograms (EOG) and a submental electromyogram. Leg movements were recorded from surface electrodes to tibialis anterior muscle of both legs. Respiratory effort was monitored from chest and abdominal respiratory inductance plethysmography bands. Sleep stages were assigned to consecutive 30 s epochs. Sleep scoring of all 20 studies was carried out by the same experienced sleep technician according to the standard rules by Rechtschaffen and Kales (1968) [8].

\subsection{RR and QT interval analysis}

The ECG signal (lead II) was band-pass filtered (0.3-30 $\mathrm{Hz}$ ), digitized at $512 \mathrm{~Hz}$ and stored for subsequent off-line analysis.

The $\mathrm{R}$ waves were automatically detected, using a signal processing algorithm. To obtain beat-to-beat QT intervals, we applied the algorithm proposed by Berger et al. (1997) [7]. Here, the operator defines a template QT interval by selecting the beginning of the QRS complex and the end of the $\mathrm{T}$ wave for one beat. The algorithm then finds the QT interval of all other beats by determining how much each beat must be stretched or compressed in time to best match the template. In this way, a relatively robust estimation of QT intervals is achieved that takes into consideration the whole $\mathrm{T}$ wave instead of commonly applied threshold techniques that are based on determining the end of the $\mathrm{T}$ wave and are therefore sensitive to artifacts and noise. For a more detailed description of the algorithm, see [7].

To assess RR and QT changes associated with spontaneous and respiratory arousal during stage 2 sleep, we automatically extracted 30 second long ECG segments starting 15 seconds prior to the onset of spontaneous or respiratory arousals, respectively, as identified in the EEG according to the standard arousal scoring rules [8]. RR and QT intervals were subsequently interpolated at $500 \mathrm{~ms}$ to obtain equidistant samples and consequently a common time base.

\subsection{Statistics}

RR, QT and PR responses for each arousal were categorized as significant shortening, lengthening or no significant change using Cusum analysis, i.e. according to departure of cumulative changes from a baseline beyond the baseline's confidence limits. The time interval 15-5 seconds prior the arousal was considered baseline.

\section{Results}

\subsection{Polysomnographic findings}

The total sleep time was $347 \pm 58 \mathrm{~min}$ and the average duration of wake periods during sleep was $63 \pm 48 \mathrm{~min}$.
The number of arousals per hour (spontaneous, respiratory and limb movement) was $26 \pm 12$. The average oxygen desaturation during respiratory events was $3.8 \pm 1.8 \%$. The mean value and range of respiratory disturbance index (RDI, number of hypopneas/apneas per hour) were $49 \pm 28$ and 17-107, respectively (normal range $<15$; 15-30 mild; 30-45 moderate; and $>45$ severe OSA according to the American Academy of Sleep Medicine Task Force (1999) [9]).

\subsection{Arousal ECG analysis}

Altogether, ECG data from 67 spontaneous and 120 respiratory arousals during stage 2 sleep were included in the analysis. Figure 1 and 2 show the group averaged RR and QT time series before and after the onset of spontaneous and respiratory arousals, respectively.

A significant number of RR intervals (64\%) shortened after arousal $(55 \%$ during spontaneous and $68 \%$ during respiratory arousal). Eighteen percent of all RR intervals were prolonged, whereas there were significantly more prolongations during spontaneous arousal (27\% during spontaneous versus $13 \%$ during respiratory arousal).

Similarly, a significant number of QT intervals (65\%) shortened after arousal (58\% during spontaneous and $68 \%$ during respiratory arousal). Only 13\% were prolonged (16\% during spontaneous and $12 \%$ after respiratory arousal).

\section{Discussion and conclusions}

Cortical arousals have been associated with changes in the RR and QT interval. In agreement with previous studies [10], our data suggest that heart rate increases during arousal. Furthermore, the RR interval graph (Fig. 1) indicates that the shortening precedes the cortical arousal, as measured in the EEG, by a few seconds. This supports the theory of a hierarchical arousal response from subcortical up to cortical levels [11]. Comparing the heart rate responses caused by spontaneous arousals with those caused by respiratory arousals, the latter ones appear to be more pronounced than those to spontaneous arousals.

Besides the changes in heart rate, cortical arousals are also associated with alterations in repolarization, as reflected in the significantly increased occurrence of QT interval shortenings. This may reflect an altered temporal pattern of neurotransmitter release in the ventricular myocardium of OSA patients. In contrast to the RR interval, we found no difference in the QT interval responses between spontaneous or respiratory arousals. A shortening in QT interval has been previously described in healthy subjects in response to auditory evoked arousals [12].

In conclusion, cortical arousals are associated with cardiac changes including heart rate and ventricular repolar- 


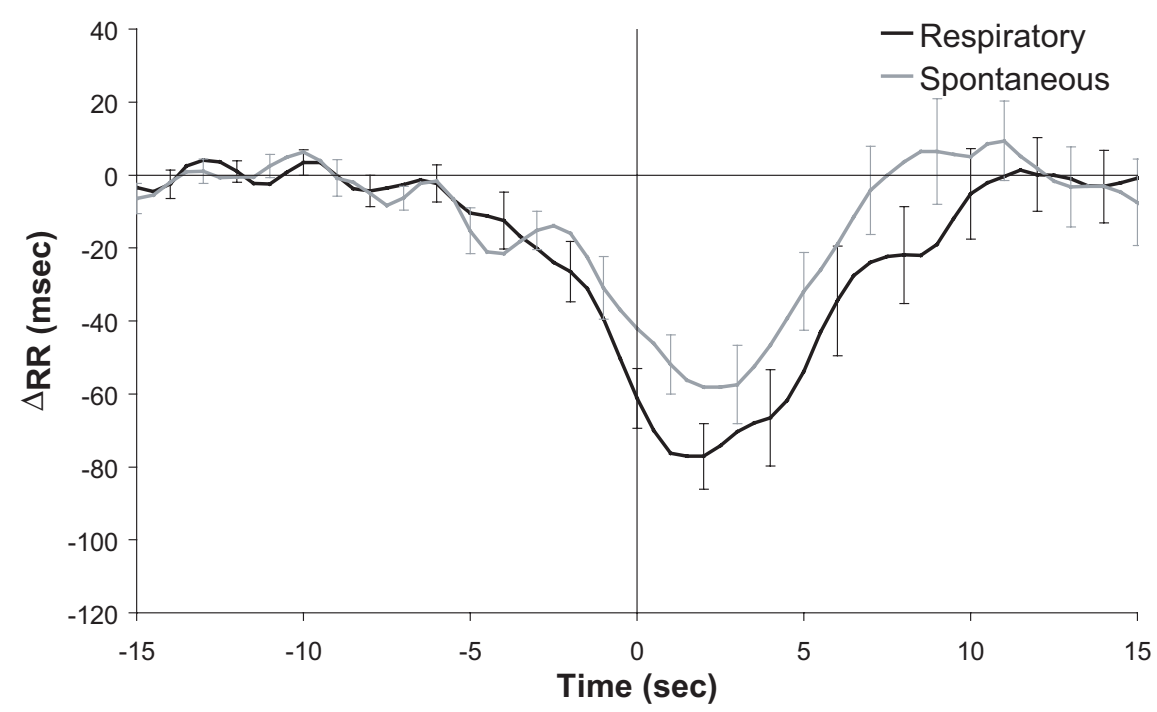

Figure 1. Averaged RR interval $\pm \mathrm{SD}$ before and after cortical arousal onset (vertical line) during stage 2 sleep.

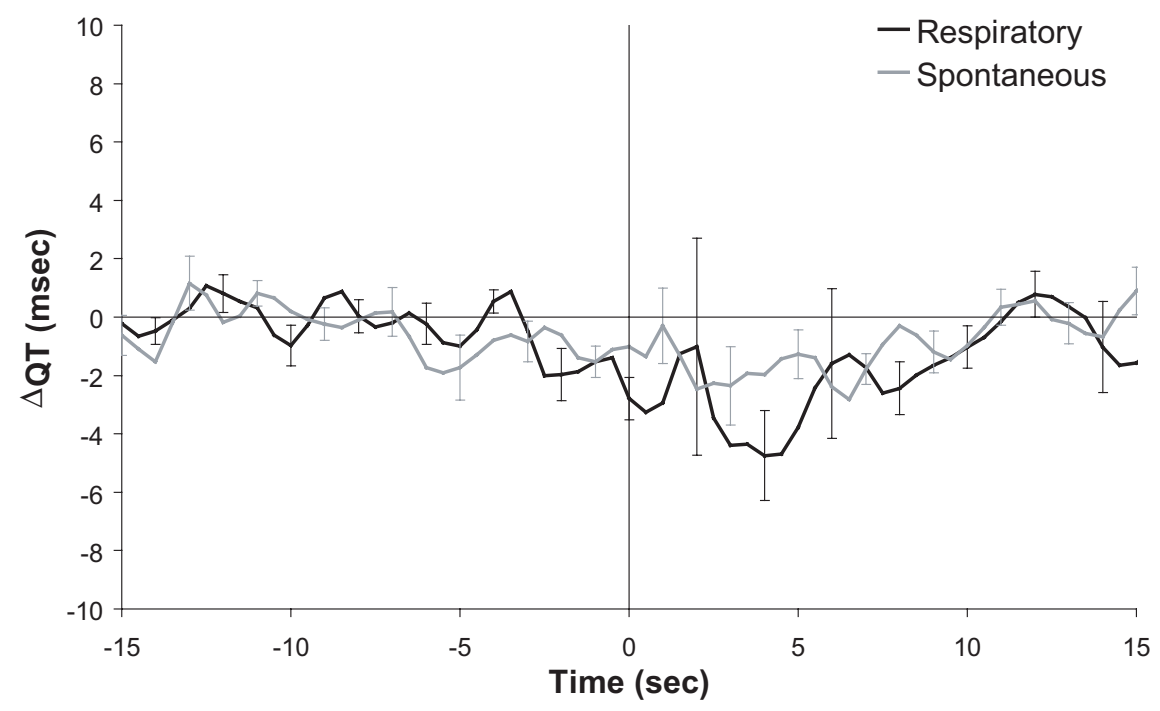

Figure 2. Averaged QT interval $\pm \mathrm{SD}$ before and after cortical arousal onset (vertical line) during stage 2 sleep.

ization. Possibly, the high prevalence of arousals in the OSA population might trigger cardiac events, perhaps explaining the increased the occurrence of nocturnal cardiac death.

\section{Acknowledgements}

The authors are grateful to Prof Ronald Berger for providing the QT analysis software and to Barry Fetics for his kind technical support. This study was partly supported by a grant from the Australian Research Council (DP0663345), the National Heart Foundation and
NH\&MRC Australia.

\section{References}

[1] Young T, Palta M, Dempsey J, et al. The Occurrence of SleepDisordered Breathing among Middle-Aged Adults. N Engl J Med 1993;328:1230-1235.

[2] Marin JM, Carrizo SJ, Kogan I. Obstructive Sleep Apnea And Acute Myocardial Infarction: Clinical Implications Of The Association. Sleep 1998;21:809-815.

[3] Guilleminault C, Connolly SJ, Winkle RA. Cardiac arrhythmia and conduction disturbances during sleep in 400 patients with sleep apnea syndrome. Am J Cardiol. 1983;52(5):490-4.

[4] Tsuji H, Larson MG, Venditti FJ, Manders ES, Evans JC, Feldman 
CL, Levy D. Impact of reduced heart rate variability on risk for cardiac events. Circulation. 1996;94:2850-2855.

[5] Sandercock GR, Brodie DA., The role of heart rate variability in prognosis for different modes of death in chronic heart failure. Pacing Clin Electrophysiol. 2006;29:892-904.

[6] Wiklund U, Olofsson BO, Franklin K, Blom H, Bjerle P, Niklasson $\mathrm{U}$. Autonomic cardiovascular regulation in patients with obstructive sleep apnoea: a study based on spectral analysis of heart rate variability. Clin Physiol. 2000;20:234-41.

[7] Berger RD, Kasper EK, Baughman KL, Marban E, Calkins H, Tomaselli GF. Beat-to-beat QT interval variability: novel evidence for repolarization lability in ischemic and nonischemic dilated cardiomyopathy. Circulation. 1997;96:1557-65.

[8] Rechtschaffen A, Kales, A (Eds.). A Manual of Standardized Terminology, Techniques and Scoring System for Sleep Stages of Human Subject. US Government Printing Office, National Institute of Health Publication, Washington DC, 1968.

[9] American Academy of Sleep Medicine Task Force. Sleep-related breathing disorders in adults: recommendations for syndrome definition and measurement techniques in clinical research. Sleep. 1999;22:667-89.
[10] Sforza E, Nicolas A, Lavigne G, Gosselin A, Petit D, Montplaisir J.EEG and cardiac activation during periodic leg movements in sleep: support for a hierarchy of arousal responses.Neurology. 1999;52:786-91.

[11] Sforza E, Jouny C, Ibanez V. Cardiac activation during arousal in humans: further evidence for hierarchy in the arousal response.Clin Neurophysiol. 2000;111:1611-9.

[12] Nalivaiko E, Catcheside PG, Adams A, Jordan AS, Eckert DJ, McEvoy RD.Cardiac changes during arousals from non-REM sleep in healthy volunteers. Am J Physiol Regul Integr Comp Physiol. 2007;292:R1320-7.

Address for correspondence:

Dr Mathias Baumert

School of Electrical \& Electronic Engineering

The University of Adelaide, SA 5005

Australia

mbaumert@eleceng.adelaide.edu.au 